\section{Nichtinvasive Positivdruck- beatmung bei akut respiratorischer Insuffizienz - Ein Report der internationalen Konsensus- konferenz der Intensivmedizin, Paris, 13. - 14. 4. 2000}

\section{Organisatorisches zur International Consensus Conference in Intensive Care Medicine}

Im Sinne der Standortbestimmung zur Bedeutung der nichtinvasiven Positivdruckbeatmung (NIV) bei akut respiratorischer Insuffizienz (ARI) fand am 13. und 14. April 2000 in Paris eine internationale Konsensuskonferenz statt. Organisiert wurde diese Veranstaltung im Sinne des „Joint meeting“ von Vertretern der American Thoracic Society (ATS), der European Respiratory Society (ERS), der European Society of Intensive Care Medicine (ESICM) und der Société de Réanimation de Loung Française (SRLF). Die der Konferenz zugrunde liegende Strategie ging auf Konzepte des National Institutes of Health [1] zurück und wurde im Weiteren den Verhältnissen der Intensivmedizin angepasst [2]. Hierbei wurden bereits vor der Konferenz die zentralen Fragen zum Stellenwert der NIV bei ARI vorformuliert. In einem weiteren Schritt folgte eine Literaturrecherche zu diesem Thema. Diese Literatur wurde einer Jury (bestehend aus international bekannten Wissenschaftlern, die keine ausgewiesene Experten auf dem Feld der NIV waren) zur Verfügung gestellt. Mitglieder der Jury waren: T. Evans (UK), F. Lemaire (Frankreich), J. Mancebo (Spanien), G. Ramsay (Niederlande), C. Richard (Frankreich), C. Sassoon (USA) und A. S. Slutsky (Kanada).

Während der Konsensuskonferenz präsentierten international renommierte Experten auf dem Gebiet der NIV bei ARI in Gegenwart der Jury und eines öffentlichen Auditoriums die Vorträge zu verschiedenen Schwerpunktthemen. Jedem Vortrag folgte eine ausführliche Diskussion zwischen Jury und Experten. Während einer Klausurtagung im unmittelbaren Anschluss an die Konsensuskonferenz wurden dann die wesentlichen Erkenntnisse unter besonderer Berücksichtigung der vorhandenen Evidenz und offen bleibender Fragen von der Jury zusammengefasst. Es ist vorgesehen, dass die wesentlichen Ergebnisse der Konferenz in Kürze in den Organen der beteiligten Gesellschaften veröffentlicht werden.

\section{Hintergrund}

Die NIV ist zur Behandlung der chronisch respiratorischen Insuffizienz (CRI) weitgehend etabliert. Der Beatmungszugang bei der NIV besteht aus einer Nasen- bzw. Nasen-MundMaske. Insbesondere für die CRI infolge thorakorestriktiver

Pneumologie 2001; 55: $21-30$

(C) Georg Thieme Verlag Stuttgart · New York ISSN 0934-8387
PD Dr. B. Schönhofer

Krankenhaus Kloster Grafschaft, Zentrum für Pneumologie, Beatmungs- und Schlafmedizin, 57392 Schmallenberg-Grafschaft und neuromuskulärer Erkrankungen ließ sich nachweisen, dass NIV zur Verbesserung von Lebensqualität und Prognose führt [3].

Bei der Behandlung der ARI steht der NIV die invasive Beatmung via Trachealtubus gegenüber. Im Gegensatz zur CVI lässt sich zur Zeit der Stellenwert der NIV als Therapieprinzip bei ARI noch nicht abschließend beurteilen.

Zentrale Fragenkomplexe der Konsensuskonferenz zur NIV bei ARI waren

Welche Rationale liegt der NIV bei ARI zugrunde?

Existieren Daten zur Epidemiologie und Pathophysiologie?

Welches Equipment und welche Beatmungsmodi sollen benutzt werden?

Wer soll NIV anwenden? Wo und wie soll NIV angewandt werden?

Welche Indikationen für NIV bestehen bei ARI? Wo liegen die Limitationen, Risiken und Kontraindikationen für NIV?

\section{Welche Rationale liegt der NIV zugrunde?}

Referenten: N. Ambrosino (Italien), P. Jolliet (Schweiz), V. Jounieaux (Belgien), C. Brunn-Buisson (Frankreich)

\section{Allgemeine pathophysiologische Betrachtung zur ARI}

ARI lässt sich aufgrund der zugrunde liegenden Pathophysiologie unterscheiden in den hyperkapnischen Typ (infolge Hypoventilation) sowie den hypoxämischen Typ. Beim hyperkapnischen Typ, z.B. dem akut dekompensierten Asthma bronchiale oder der exazerbierten COPD geht es um die Reduktion der Hyperkapnie, Stabilisierung des arteriellen pH und Entlastung der Atemmuskulatur. Die Zielgröße der NIV bei hypoxischem ARI ist die Verbesserung der Oxygenierung. Bei dem kardial bedingten Lungenödem wird darüber hinaus die Verbesserung der Hämodynamik und der Diurese angestrebt.

\section{NIV versus invasive Beatmung}

Es besteht ein Spannungsfeld zwischen Vor- und Nachteilen der NIV im Vergleich zur invasiven Beatmung. Die wesentlichen Vorteile der NIV sind die Vermeidung der endotrachea- 
len Intubation und der hiermit assoziierten Komplikationen, die Reduktion oder sogar der weitgehende Verzicht auf Sedierung, die Reduktion der Verweildauer auf der Intensivstation bzw. im Krankenhaus. Mögliche Nachteile der NIV können die subjektive Beeinträchtigung durch die Maske, der unsichere Beatmungszugang, der unzureichende Gasaustausch, die vermehrten Kosten durch Training und Ausbildung der Mitarbeiter bzw. erhöhter Aufwand für das Personal sein.

\section{Epidemiologie und Zielgruppe für NIV}

Kürzlich ließ sich für Großbritannien zeigen, dass etwa $20 \%$ der hospitalisierten Patienten mit chronisch obstruktiver Lungenerkrankung (COPD) mögliche Kandidaten für die NIV sind [4]. Im Gegensatz zu dieser Einschätzung ergab eine französische multizentrische Studie bei 42 Intensivstationen, dass NIV „im wirklichen Leben“ nur in 16\% der Fälle (Range 0-67\%) als primäre Beatmungsform genutzt wurde [5]. Ernüchternd ergab des weiteren eine Studie, die an englischen Krankenhäusern durchgeführt wurde, dass 52\% der Krankenhäuser keine Erfahrung mit bzw. Kapazitäten für NIV bei ARI hatten [6] und 68\% der Krankenhäuser mit „NIV-Erfahrung“ weniger als 20 Patienten pro Jahr mit dieser Therapie behandelten.

\section{Atmungsphysiologische Effekte der NIV bei ARI}

Sowohl physiologische als auch klinische Studien ergaben für die hyperkapnische Verlaufsform der ARI (z.B. bei „acute on chronic respiratory failure“ bzw. Exazerbation der COPD), dass unterschiedliche Beatmungsverfahren, z.B. „assist-control ventilation“ (ACV) [7] „pressure support ventilation“ (PSV) [8-10] bzw. „proportional assist ventilation“ (PAV) [11] zu einer Verbesserung des Atemmusters $[8,10,11]$, der Dyspnoe [12-14] und der Blutgase $[8,10,11,13-15]$ führen. Des Weiteren kommt es infolge NIV zur Entlastung der Atemmuskulatur $[8,10,16]$ sowie durch externe Applikation von positive endexpiratory pressure (PEEP) zur Reduktion des intrinsischen PEEP [8].

Des Weiteren ließ sich für die hypoxämische Verlaufsform der ARI in Folge unterschiedlicher Ursachen zeigen, dass es durch NIV zur Verbesserung der Blutgase und der Dyspnoe, zur Reduktion der Atemfrequenz sowie zur Entlastung der Atemhilfsmuskulatur kommt $[17,18]$.

Schließlich ergab sich für das akut auftretende kardial bedingte Lungenödem, dass sowohl CPAP als auch inspiratorische Druckunterstützung zur Abnahme von Atemfrequenz und respiratorischer Azidose [19-21] führen.

\section{Hämodynamische Effekte der NIV}

$\mathrm{Zu}$ dieser Thematik erfolgten bisher nur wenige systematische Untersuchungen. Dennoch lassen sich folgende Erkenntnisse zusammenfassen: Bei ARI ohne linksventrikuläre Insuffizienz bzw. fehlender pulmonaler Vorerkrankung hat NIV wenig negativen Einfluss auf die Hämodynamik. Allgemein gültige Daten zum kardialen Output liegen nicht vor. Beim kardial bedingten Lungenödem kommt es unter NIV zur Abnahme der Herzfrequenz, Verbesserung der linksventrikulären Funktion und geringer Abnahme des systemischen arteriellen Blutdrucks [19,20,22].
Sollte jedoch bei vorliegender Rechtsherzinsuffizienz bzw. bei manifester pulmonal arterieller Hypertonie auf hohe Beatmungsdrücke bzw. hohe externe PEEP-Werte verzichtet werden. Hierbei kann es infolge einer möglicherweise übermäßigen Lungenüberblähung zur Reduktion des venösen Rückflusses zum rechten Herzen und damit zur Kreislaufdepression kommen.

\section{Die Bedeutung der oberen Atemwege bei NIV}

Da die oberen Atemwege bei der NIV - im Unterschied zur invasiven Beatmung via Trachealtubus - nicht überbrückt werden, kommt insbesondere der Glottis als eigenständigem und variablem Widerstand der Atemwege eine besondere Bedeutung zu. Aufgrund des erhöhten Widerstandes der oberen Atemwege bei verengten Stimmlippen kann es zur Beeinträchtigung der Beatmungsqualität infolge Mundleckagen kommen [23,24]. Obwohl diese Erkenntnis bei gesunden Probanden ermittelt wurde, ist anzunehmen, dass sie auch von relevanter Bedeutung für die NIV bei ARI ist. Allerdings bleibt zur Zeit offen, welche Konsequenzen sich hieraus für die Einstellung des Beatmungsgerätes ergeben. Im Falle von Mundleckagen bei angelegter Nasenmaske sollte jedoch auf die Ganzgesichtsmaske umgestellt werden (siehe Abschnitt „Beatmungszugang“).

\section{Die Bedeutung der NIV auf die infektbedingten Komplikationen}

Eine kürzlich veröffentlichte Metaanalyse von evidenzbasierten Studien zur Bedeutung der NIV bei ARI ergab, dass NIV abhängig von der zugrundeliegenden Ursache der ARI - zu einer reduzierten Mortalitätsrate, Intubationsfrequenz bzw. Dauer der Beatmung und des Aufenthalts auf der Intensivstation führt [25]. Bezüglich der Rate nosokomialer Infektionen und insbesondere der ventilator-assoziierten Pneumonien (VAP) lässt sich aufgrund der vorliegenden randomisierten kontrollierten Studien ableiten, dass diese Komplikationsrate unter NIV abnimmt. Analog hierzu ließ sich in einer Studie zum Stellenwert der sich unmittelbar an eine Kurzzeitbeatmung anschließende NIV zeigen, dass auch hier die Infektionsrate reduziert ist [26]. Ein wesentlicher Mechanismus der positiven Auswirkung der NIV auf die nosokomiale Infektionsrate besteht im Vergleich zur invasiven Beatmung offensichtlich in direktem Zusammenhang mit der kürzeren Beatmungsdauer, dem kürzeren Aufenthalt auf der Intensivstation sowie der hiermit verbundenen Abnahme anderer invasiver Maßnahmen.

\section{Limitationen der Studien}

Die Ergebnisse der vorhandenen randomisierten kontrollierten Studien werden relativiert durch methodische Mängel, die die Aussagefähigkeit bzgl. der angestrebten Evidenz einschränken:

1. Entsprechend der Ausschlusskriterien wurde in den randomisierten Studien ein selektioniertes Patientenkollektiv untersucht.

2. Die untersuchten Patientenkollektive sind oft klein und heterogen und

3. in unterschiedlichen Subgruppen der Studien kann es zu unbeobachteten negativen Effekten der NIV kommen (z.B. erhöhte Mortalität). 
4. In Folge „nicht blindem Designs“ der Studien, ist eine „hyperprotektive“ Betreuung der Studienpatienten durchaus denkbar.

5. Die meisten Studien wurden in einzelnen Zentren für NIV mit hoher Erfahrung und Motivation durchgeführt. Die beiden letztgenannten Aspekte können zur falsch günstigen Interpretation der Messergebnisse führen.

\section{Zusammenfassung}

Entsprechend der zugrunde liegenden Pathophysiologie ist NIV sowohl bei hyperkapnischer als auch hypoxämischer Verlaufsform der ARI durchführbar.

Die physiologische Rationale für NIV bei ARI infolge akut exazerbierter COPD liegt vor allem in der inspiratorischen Unterstützung der Atemmuskulatur, dem verbesserten Gasaustausch sowie der Antagonisierung des intrinsischen PEEP durch Applikation eines externen PEEP.

Während NIV ist eine negative Auswirkung auf die Hämodynamik nur in Ausnahmefällen zu erwarten.

Eine Erhöhung des Atemwegswiderstandes infolge funktionell verengter Glottis kann zur Beeinträchtigung der NIV-Qualität führen.

Im Vergleich zu invasiver Beatmung führt NIV bei ARI zur deutlichen Reduktion der VAP.

\section{Empfehlungen für zukünftige Studien}

Um das pathophysiologische Verständnis für den Stellenwert der NIV bei hypoxämischer ARI zu verbessern, sind Studien zur Effektivität der NIV bei z.B. Patienten mit Pneumonie, ARDS (adult respiratory distress syndrome) oder traumatischer Lungenschädigung notwendig.

Des Weiteren muss die Bedeutung der maschinellen Unterstützung der Atemmuskulatur versus externem PEEP in der Behandlung unterschiedlicher Formen der ARI untersucht werden.

Es werden Untersuchungen zur Ermittlung prädiktiver Parameter bzgl. Outcome und Responderrate nach NIV bei ARI benötigt.

\section{Welches Equipment und welche Beatmungsmodi sollten verwandt werden?}

Referenten: P. Gay (USA), F. Lofasa (Frankreich), S. Nava (Italien), D. Robert (Frankreich), A. Rossi (Italien) und M. Vitacca (Italien)

\section{Ventilatortypen}

Abhängig vom Schweregrad der Erkrankung, von der jeweiligen Lokalität und der Erfahrung des behandelnden Teams werden zur Durchführung der NIV bei ARI sowohl portable „Heimbeatmungsgeräte“ als auch komplizierte „Intensivrespiratoren" verwendet. Auch wenn sich beide Gerätetypen zumindest bezüglich des „Pressure Support“-Modus ähneln, bestehen doch wesentliche Unterschiede bezüglich techni-
Tab. 1 Vergleich zwischen Intensivventilatoren und portablen (Heimbeatmungs-)ventilatoren

\begin{tabular}{|c|c|c|}
\hline Aspekte & Intensivventilatoren & portable Ventilatoren \\
\hline Technik & hochwertig & relativ einfach \\
\hline $\begin{array}{l}\text { Monitoring } \\
\text { und Alarme }\end{array}$ & hochwertig & $\begin{array}{l}\text { nicht vorhanden } \\
\text { oder minimiert }\end{array}$ \\
\hline Kommentar & $\begin{array}{l}\text { nicht jeder Ventilator } \\
\text { ist für NIV bei ARI } \\
\text { geeignet }\end{array}$ & $\begin{array}{l}\text { trotz relativ einfacher } \\
\text { Technik ist häufig eine } \\
\text { adäquate NIV bei ARI } \\
\text { möglich }\end{array}$ \\
\hline
\end{tabular}

scher Details (Tab.1). Im Gegensatz zu den vergleichbar einfach gehaltenen portablen Beatmungsgeräten befindet sich die Technik der Intensivrespiratoren auf hohem Niveau. Es ließ sich dennoch zeigen, dass portable Ventilatoren, trotz simpler Technik, bezüglich den allgemeinen Anforderungen an Ventilatoren zur Therapie von ARI den Intensivrespiratoren durchaus vergleichbar sind [27]. Bezüglich des Monitorings verfügen die Intensivrespiratoren obligat über die kontinuierliche Messung von $\mathrm{FiO}_{2}$, Tidal-Volumen, Atemfrequenz und Atemminutenvolumen. Darüber hinaus erfolgt die Quantifizierung der Leckagen (über Mund oder Maske) und die visualisierte Darstellung von Fluss- und Druckkurven. Auch wenn hierzu keine Studienergebnisse vorliegen, sind Monitoring und Alarme insbesondere bei schwergradig ausgeprägter ARI (z.B. $\mathrm{pH}<7,2$ ) erforderlich.

\section{Beatmungsverfahren}

Im Prinzip stehen bei NIV-Beatmungsverfahren mit Druckbzw. Volumenvorgabe im assistierten, assistiertkontrollierten oder kontrollierten Modus zur Verfügung.

Vorausgesetzt, dass ein Patient unbeeinträchtigt spontan atmen kann, ist in einigen Untergruppen der ARI (z.B. beim kardial bedingten Lungenödem, siehe dort) „continuous positive airway pressure“ (CPAP) ein probater Modus. Der am häufigsten zur Behandlung der ARI eingesetzte Modus ist jedoch „Pressure Support Ventilation“ (PSV). Optional wird bei den Bi-Level-Ventilatoren neben PSV zusätzlich externer PEEP (= CPAP) verwandt. Die angestrebten Vorteile von PSV bzw. dem Bi-Level-Mode bestehen in der Leckagekompensation, Verbesserung der Patienten-Ventilator-Synchronität (d.h. im Idealfall adäquater Trigger, regelrechte Adaptation an In- und Exspiration des Patienten sowie Reduktion der dynamischen Überblähung bei Patienten mit tracheobronchialem Kollaps bzw. intrinsischem PEEP).

Trotz der genannten Vorteile lassen sich vier Bereiche benennen, bei denen es prinzipiell zur Dysfunktion des PSV-Mode kommen kann: inspiratorischer Trigger [28], Druckaufbau [29], expiratorischer Trigger [30] und $\mathrm{CO}_{2}$-Rückatmung [31]. In Tab. 2 werden die zugrunde liegenden Ursachen für die Problematik sowie mögliche Lösungskonzepte aufgeführt.

Einen Subtyp der PSV stellt die Proportional-Assist-Ventilation (PAV) dar. Hierbei kommt es synchronisiert zum Atemmuster des Patienten zu einer Druckunterstützung der Inspiration proportional zum Atemantrieb des Patienten [32]. Es 


\begin{tabular}{|c|c|c|}
\hline technisches Problem & Ursachen & Lösungsansätze \\
\hline $\begin{array}{l}\text { inspiratorische Trigger } \\
\text { (inadäquate Detektion } \\
\text { der Inspiration) }\end{array}$ & $\begin{array}{l}\text { 1. Leckage } \\
\text { 2. „Auto-Cycling“ } \\
\text { 3. Drucktriggerung }\end{array}$ & $\begin{array}{l}\text { 1. Nasen-Mund-Maske } \\
\text { 2. schnelle Reaktion auf variable } \\
\text { Flussrate } \\
\text { 3. Flowtriggerung }\end{array}$ \\
\hline $\begin{array}{l}\text { Druck-Fluss-Entwicklung } \\
\text { (Inadäquate inspiratorische } \\
\text { Flussrate) }\end{array}$ & $\begin{array}{l}\text { 1. zu träge Druckanstiegszeit } \\
\text { 2. zu geringe Druckunter- } \\
\text { stützung }\end{array}$ & $\begin{array}{l}\text { 1. kürzere Druckanstiegszeit } \\
\text { 2. höhere Druckunterstützung }\end{array}$ \\
\hline $\begin{array}{l}\text { exspiratorischer Trigger } \\
\text { (inadäquate Erfassung der } \\
\text { dezelerierenden Flussrate) }\end{array}$ & Leakage & $\begin{array}{l}\text { 1. Nasen-Mund-Maske } \\
\text { 2. vorgegebene Maximaldauer } \\
\text { der Inspiration }\end{array}$ \\
\hline $\mathrm{CO}_{2}$-Rückatmung & $\begin{array}{l}\text { 1. Einschlauchsystem } \\
\text { (kein Exspirationsventil) } \\
\text { 2. hohe Atemfrequenz } \\
\text { 3. zu geringer PEEP }\end{array}$ & $\begin{array}{l}\text { 1. Zweischlauchsystem, Exspira- } \\
\text { tionsventil } \\
\text { 2. Reduktion der Atemfrequenz } \\
\text { 3. höherer PEEP }\end{array}$ \\
\hline
\end{tabular}

Tab. 2 Pressure support ventilation (PSV): technische Probleme dieses Modus, Ursachen hierfür und mögliche Lösungsansätze ließ sich zeigen, dass ein wesentlicher Wirkmechanismus der PAV die Entlastung der Atemmuskulatur ist [33]. Bezüglich des Stellenwertes von PAV für die NIV bei ARI bleibt festzustellen, dass hierzu bisher nur wenige Studien mit geringen Patientenzahlen existieren $[11,18,34]$ und sich PAV in diesem Zusammenhang im experimentellen Stadium befindet. Bisher ergibt sich bezüglich physiologischer Parameter kein eindeutiger Vorteil von PAV gegenüber PSV. Möglicherweise ist mit der Anwendung von PAV ein größerer Komfort für den Patienten verbunden.

Nur in wenigen Studien wurde ein direkter Vergleich zwischen Beatmungsverfahren mit Druck- bzw. Volumenvorgabe durchgeführt. In diesen Studien erfolgt die Beatmung mit Volumenvorgabe im „Assist-Controlled-Ventilation-Mode, (ACV) [10,35-37]. Ein Vergleich zwischen PSV und ACV bezüglich einiger physiologischer, aber auch klinischer Aspekte, ist in Tab. 3 aufgeführt.

Auch wenn kontrollierte Beatmung bei NIV zur Behandlung der ARI nur selten eingesetzt wird, kann sie jedoch bei bestimmten Indikationen von Vorteil sein. Hierzu gehören eine massive Überlastung der Atemmuskulatur, unzureichender Atemantrieb bzw. Hypoventilation, leckagebedingt unphysiologisch verlängerte Inspirationszeit und Versagen von assistierten Beatmungsformen.

Tab. 3 Vergleich zwischen Pressure support ventilation (PSV) und assistiertkontrollierter Beatmung (ACV) mit Volumenvorgabe; +: Vorteil, -: Nachteil

\begin{tabular}{lll}
\hline & PSV & ACV \\
\hline Beatmungsqualität & + & + \\
Gasaustausch & + & + \\
klinischer „Outcome“ & + & + \\
Patientenkomfort & + & - \\
Leakagekompensation & + & - \\
autonome Atmungsregulation & + & - \\
Reduktion der Atemarbeit & $(-)$ & + \\
\hline
\end{tabular}

Abschließend soll nicht unerwähnt bleiben, dass in jüngerer Vergangenheit von einigen Herstellern Beatmungsgeräte entwickelt wurden, die eine Kombination der Beatmung mit Volumen- bzw. Druckvorgabe ermöglichen. Obwohl diese Entwicklung durchaus vielversprechend erscheint, liegen hierzu bisher keine wissenschaftlich gesicherten Daten vor.

\section{Beatmungszugang (Interface)}

Basierend auf einer Medline-Literaturrecherche bzgl. des Beatmungszuganges ergab sich folgende Anwendungsfrequenz: Ganzgesichtsmaske (63\%), nasale Maske (31\%) und Nasenoliven (6\%); demgegenüber werden Mundstücke (Mouth-pieces) sowie individuell hergestellte Masken nur in seltenen Fällen angewandt. Entsprechend einer Abwägung von Vor- und Nachteilen wird die Ganzgesichtsmaske bei der Behandlung der ARI gegenüber der Nasenmaske als Beatmungszugang bevorzugt (siehe Tab.4). Die wesentlichen

Tab. 4 Vergleich zwischen Nasen-Mund-Maske und Nasenmaske als Beatmungszugang bei akut respiratorischer Insuffizienz; +: Vorteil, -: Nachteil

\begin{tabular}{lll}
\hline klinischer Aspekt & $\begin{array}{l}\text { Nasen-Mund- } \\
\text { Maske }\end{array}$ & $\begin{array}{l}\text { Nasen- } \\
\text { maske }\end{array}$ \\
\hline Mundleakage & + & - \\
Mundatmung & + & - \\
Monitoring des Atemzugvolumens & + & - \\
Beeinträchtigung Nasenatmung & + & - \\
Einfluss des Zahnstatus & + & - \\
hohe Beatmungsdrücke & + & - \\
Dynamik der gebesserten Blutgase & + & - \\
Komfort und Toleranz & - & + \\
Kommunikationsfähigkeit & - & + \\
Nahrungsaufnahme & - & + \\
Exspektoration & - & + \\
Aspirationsgefahr & - & + \\
Aerophagie & - & + \\
Klaustrophobie & - & + \\
Totraum & $(-)$ & \\
\hline
\end{tabular}


Vorteile der Ganzgesichtsmaske bestehen in der Kompensation von Mundleckagen, einer während der NIV weiterhin möglichen Mundatmung sowie der Möglichkeit des Monitorings des Tidal-Volumens.

Unabhängig vom verwandten Maskentyp stellen die maskeninduzierten Druckstellen bzw. Läsionen des Nasenrückens ein relevantes Problem dar. Unterschiedliche Strategien tragen zur Verbesserung des Maskensitzes und Verhinderung der Läsionen bei: Es können Materialien zum Schutz der Haut auf den Nasenrücken aufgebracht werden. Darüber hinaus sind Masken mit Gelmaterial in Gebrauch. Außerdem sind Masken verfügbar, deren Sitz durch Ausspritzen der Maskenrandregion mit Kunststoffen (z.B. Silikon) der Gesichtsform individuell angepasst werden können („Semi-customised mask“). Schließlich lassen sich im Sinne der Ultima ratio komplette Masken binnen kurzer Zeit individuell anfertigen.

\section{Monitoring}

Ein wesentlicher Bestandteil des Monitorings besteht in der klinischen Beobachtung des Patienten. So sind z.B. der Grad der Agitation, der Dyspnoe, des Komforts, die Atemfrequenz, die Atemanstrengung, der Einsatz der Atemhilfsmuskulatur, das Atemmuster (z.B. paradoxe Atmung) oder vegetative Symptome durch Inspektion zu erfassen.

Darüber hinaus ist jedoch ein polyfunktionelles apparatives Monitoring mittels kontinuierlicher Messung von quantifizierbaren Parametern der Vitalfunktionen (wie z.B. Atemfrequenz, Tidal-Volumen, Atemminutenvolumen und EKG) unverzichtbar. Des Weiteren besteht ein wesentlicher Bestandteil des nichtinvasiven Monitorings in der pulsoximetrisch ermittelten Sauerstoffsättigung, die gewisse Aussagen zur Effektivität der NIV bzw. Oxygenierung zulässt. Vor allem in der Initialphase der NIV bei ARI müssen zusätzlich engmaschig durchgeführte Blutgasanalysen zur Ermittlung des pHWertes - bzw. der $\mathrm{PCO}_{2}$-Werte - erfolgen. Optional kann das nichtinvasive Monitoring der NIV durch das transkutan oder endtidal gemessene $\mathrm{CO}_{2}$ ergänzt werden. In seltenen Einzelfällen ist es sinnvoll, das nichtinvasive Monitoring durch ein invasives Monitoring in Form des arteriellen Zugangs und des Pulmonaliskatheters zu ergänzen.

Vor allem in der Initialphase der NIV ermöglicht nur die gleichzeitige Analyse mehrerer Parameter im Sinne des multifunktionellen Monitorings eine adäquate Verlaufsbeobachtung der NIV. NIV-Versagen ist charakterisiert durch weitere Verschlechterung der Blutgasanalyse (d.h. insbesondere des pH-Wertes) innerhalb der ersten Stunden nach Beginn der NIV, Veränderungen des Atemmusters im Sinne einer $\mathrm{Zu}$ nahme der Atemfrequenz und Abnahme des Tidal-Volumens, zunehmende hämodynamische Instabilität oder Zeichen der Vigilanzstörung.

\section{Zusammenfassung}

Es besteht keine Evidenz zur bevorzugten Verwendung bestimmter Beatmungsgeräte, Beatmungsmodi oder Interfaces.

Die konkrete Wahl des Beatmungsgerätes bzw. -modus ist im Wesentlichen abhängig von der Erfahrung und Schulung des behandelnden Teams. Dennoch lässt sich festhalten, dass PSV häufig kombiniert mit externem PEEP das dominierende Beatmungsverfahren zur Therapie der ARI ist.

Die konkrete Geräteeinstellung erfolgt nach mehreren Gesichtspunkten: Patientenkomfort, Entlastung der Atemmuskulatur sowie Verbesserung des Gasaustausches. Der Beatmungstyp sowie das Niveau des Monitorings sollte sich nach dem Schweregrad der Erkrankung des Patienten sowie den örtlichen Gegebenheiten richten.

Die klinische Erfahrung spricht dafür, dass Ganzgesichtsmasken vor allem wegen der Reduktion von Mundleckagen im Vergleich zu vor allem Nasenmasken in der Behandlung der ARI von Vorteil sind.

Im Sinne der optimierten Patientenbetreuung muss ein breites Sortiment an unterschiedlichen Masken verfügbar sein.

\section{Empfehlung für zukünftige Studien}

Der differenzierte Einsatz verschiedener Beatmungsgeräte und -modi in Abhängigkeit von der zugrundeliegenden Pathophysiologie der ARI muss weiter untersucht werden.

Die Entwicklung von Beatmungsgeräten, die eine optimale ventilatorische Unterstützung entsprechend des individuellen Atemmusters der Patienten (z.B. auch automatische Adaptation an individuelle Atemmuster) ermöglichen, ist wünschenswert. Studien zur Erstellung von Empfehlungen zur optimalen Einstellung der Beatmung bzgl. Beatmungsdrucke und Tidal-Volumina während der Adaptationsphase sind erforderlich.

Vergleichsstudien zwischen verschiedenen Maskentypen werden benötigt.

\section{Wer wendet NIV an? Wo und wie wird NIV durchgeführt?}

Referenten: J. C. Chevrolet (Schweiz), G. Criner (USA), M. Elliott (UK), J. C. Richard (Frankreich), B. Schönhofer (Deutschland), J. A. Wedzicha (UK), M. Wysocki (Frankreich)

Es hängt von vielen spezifischen Faktoren ab, wer für die Adaptation und Überwachung der NIV bei Patienten mit ARI verantwortlich ist. Fest steht, dass das zuständige Personal eine fundierte Schulung benötigt und große Erfahrung bei der Anwendung von NIV braucht, um eine optimale Adaptation gewährleisten zu können. Der Implementation der NIV bei ARI muss höchste Aufmerksamkeit zukommen, da sie einen sehr kritischen Zeitraum in der Betreuung des Patienten darstellt. Es ist erstaunlich, dass auch zu dieser Thematik trotz ihrer hohen Relevanz kaum durch Studien gesicherte Daten vorliegen.

In einer kürzlich veröffentlichten multizentrischen Studie aus Frankreich wurde nachgewiesen, dass die Rate des NIVVersagens wesentlich vom Schweregrad der Erkrankung und der patientenseitigen Therapieakzeptanz abhängig ist [5]. Analog hierzu wiesen Soo-Hoo et al. für ein kleineres Patientenkollektiv nach, dass die NIV-Intoleranz im Wesentlichen durch unzureichendes Maskenmaterial verursacht wurde [38]. Diese Studien lassen den Schluss zu, dass gerade 
während der Adaptationsphase große Erfahrung und besonderer Aufwand (insbesondere bzgl. der Auswahl der Maske, des Beatmungsverfahrens und der Beatmungseinstellung) erforderlich sind, um eine hohe Akzeptanzrate des Patienten für die NIV zu gewährleisten.

In dem Zusammenhang soll noch einmal erwähnt werden, dass die meisten Studien zum Thema NIV bei ARI von geschultem und hochmotiviertem Personal durchgeführt wurden. Vice versa bedeutet dies, dass aufgrund der häufig guten Therapieergebnisse der Literatur keine allgemeingültigen Schlüsse gezogen werden können.

\section{Arbeitszeitbelastung durch NIV}

Die ersten Stunden der NIV-Adaptation stellen die kritische Phase dieser Therapieform dar. Geschultes Personal (d.h. Pflegekräfte, spezialisierte MTA oder Ärzte) muss sich in dieser Phase häufig im Verhältnis 1:1 mit dem Patienten beschäftigen.

In der Vergangenheit untersuchten mehrere Studien den mit NIV einhergehenden Zeitaufwand bei ARI. Eine bereits früh erschienene unkontrollierte Studie ergab, dass NIV einen übermäßigen Zeitaufwand benötigt [39]. Spätere Studien ergaben jedoch, dass NIV im Vergleich zur konventionellen Therapie (d.h. auch der invasiven Beatmung) während der ersten Stunden für ein trainiertes Team keinen vermehrten Zeitaufwand darstellt $[13,14,40]$. Es kam in der NIV-Gruppe während der folgenden Zeitphase für die betreuende Pflegekraft sogar zu einem geringeren Zeitaufwand [41].

\section{Ort des Geschehens}

Die Wahl des Bereichs im Krankenhaus, in dem NIV bei ARI durchgeführt wird, ist von vielen Faktoren abhängig. Hierzu gehören die Qualität der Schulung und das Ausmaß der Erfahrung des behandelnden Teams, die verfügbare Technik und weitere Ressourcen (z.B. Betten, Personalschlüssel und Monitoring) und nicht zuletzt die Ätiologie der ARI bzw. der Schwergrad der Erkrankung des Patienten. Anhand der Literatur wurde NIV bei ARI bereits in den folgenden Bereichen durchgeführt: Intensivstation [9], Notfallraum [42], Intermediate-Care-Station $[37,43]$ und Normalstation [44]. In diesem Zusammenhang besonders erwähnenswert ist eine kürzlich erschienene multizentrische Studie aus England, die NIV bei akut exazerbierter COPD (Einschlusskriterien: pH 7,25-7,35, $\mathrm{PCO}_{2}>45 \mathrm{~mm} \mathrm{Hg}$, Atemfrequenz $>23 / \mathrm{min}$ ) auf Normalstation durchführt. NIV wurde mit einem Heimbeatmungsgerät und fixer Beatmungseinstellung (d.h. IPAP $15 \mathrm{~cm} \mathrm{H} \mathrm{H}_{2} \mathrm{O}$, EPAP $5 \mathrm{~cm}$ $\mathrm{H}_{2} \mathrm{O}$ ) von trainierten Pflegekräften durchgeführt. Zentrales Ergebnis war, dass es durch frühzeitige Anwendung der NIV im Vergleich zur medikamentös behandelten Therapiegruppe insbesondere auch außerhalb der Intensivstation zur Reduktion der Intubationsfrequenz sowie der Krankenhausmortalität kommt. Allerdings ergab die Subgruppenanalyse eine Verschlechterung der Prognose, wenn der $\mathrm{pH}$-Wert vier Stunden nach Anwendung von NIV weiterhin < 7,30 war. Es zeigte sich in einer weiteren Studie, dass die Verzögerung der endotrachealen Intubation nach NIV-Versagen bei ARI eine Verschlechterung der Prognose zur Folge haben kann [42]. Aus diesen Ergebnissen lässt sich schließen, dass bei Anwendung der NIV bei ARI außerhalb der Intensivstation eine im
Bedarfsfall schnelle endotracheale Intubation sowie unmittelbare Verlegung auf eine nahegelegene Intensivstation möglich sein muss.

\section{Zusammenfassung}

Der Adaptationsphase kommt bzgl. Erfolgsrate der NIV bei ARI eine entscheidende Schlüsselrolle zu. Nur geschultes Personal mit entsprechender Erfahrung gewährleistet eine optimale Adaptation des Patienten. Infolge defizitärer Implementation der NIV ist die Non-Responderrate hoch.

In einem erfahrenen Team erfordert die NIV im Vergleich zur invasiven Beatmung keinen höheren Zeitaufwand. Abhängig von den örtlichen Gegebenheiten, dem Stand der Schulung und dem Schweregrad der Erkrankung des Patienten kann NIV bei ARI in der Intensivstation, im Notfallraum, in der Intermediate-Care-Station oder sogar auf der Normalstation durchgeführt werden.

Zunächst bleibt die Intensivstation jedoch der bevorzugte Bereich, um NIV bei ARI durchzuführen. Wenn NIV außerhalb der Intensivstation angewandt wird, muss das Personal ausgebildet, ein Monitoring auf relativ hohem Niveau vorhanden sowie eine unmittelbare Intubation und sofortige Verlegung auf die Intensivstation gewährleistet sein.

\section{Empfehlung für zukünftige Studien}

Kriterien zur Verlegung von Patienten mit ARI auf die Intensivstation und zur endotrachealen Intubation nach NIVVersagen müssen definiert werden.

Es sind direkte Vergleichsstudien zwischen unterschiedlichen Lokalitäten zur Durchführung der NIV notwendig.

Untersuchungen zur Bedeutung der Qualifikation, Art der Schulung sowie zum Zeitaufwand für das Personal in nicht spezialisierten Abteilungen sind notwendig. Es fehlen internationale Vergleichsstudien zu diesen Aspekten, da sich der Pflegestandard und die Organisation der Abteilungen in verschiedenen Ländern deutlich unterscheiden.

Es muss des Weiteren abgeklärt werden, ob NIV auf der Normalstation im Sinne eines „pflegerischen“ Steal effects auf Kosten anderer Patienten geht.

Kriterien zur Beendigung der NIV werden benötigt.

\section{Bei welcher klinischen Indikation wird NIV eingesetzt?}

Referenten: A. Agustí (Spanien), M. Antonelli (Italien), C. Girauld (Frankreich), J. Mancebo (Spanien), U. Meduri (USA), S. Mehta (Kanada), J. F. Muir (Frankreich) und S. Nava (Italien)

Allgemein akzeptierte Kontraindikationen für die NIV sind in Tab. 5 aufgeführt. Zentrale Zielgrößen der NIV bei ARI sind neben der Verbesserung physiologischer Parameter (z. B. Gasaustausch, Dyspnoe, Überlastung der Atemmuskulatur, hämodynamischer Status) bestimmte Outcomeparameter (z. B. Intubationsfrequenz, Komplikationen wie nosokomiale Infekte, Aufenthalt auf der Intensivstation sowie im Krankenhaus, Kosten und Prognose). 
Tab. 5 Absolute Kontraindikation für NIV bei akut respiratorischer Insuffizienz

schwergradige Azidose bei Aufnahme $(\mathrm{pH}<7,1)$

lebensbedrohliche und therapierefraktäre Hypoxämie

längerer Atemstillstand und fehlender Atemantrieb

Koma oder Konfusion und fehlende Kooperation

schwergradige psychomotorische Agitation

relevante Komorbidität (z. B. Mehrorganversagen)

orofaziale Dysmorphie

Zustand nach akut durchgeführtem gesichtschirurgischem Eingriff

vollständige Maskenintoleranz

hämodynamische Instabilität, Schock

(systolischer Blutdruck $<70 \mathrm{~mm} \mathrm{Hg}$ )

schwergradige Hypersekretion

(d.h. Bronchoskopie mehrfach täglich notwendig)

hohe Aspirationsgefahr

notwendige Protektion der oberen Atemwege

(z. B. bei Koma und akutem abdominalen Prozess)

Glottisödem oder andere Ursachen einer relevanten Glottisobstruktion

\section{Hyperkapnische Verlaufsform der ARI}

In mehreren randomisierten Studien ließ sich für den hyperkapnischen Typ der ARI im Vergleich zur konventionellen Therapie weitgehend konsistent zeigen, dass sich vor allem bei Patienten mit akut exazerbierter COPD durch NIV eine Besserung der Vitalfunktion, Reduktion der Intubationsfrequenz, Abnahme der Komplikationsrate, Abnahme des Krankenhausaufenthalts sowie Mortalität innerhalb des Krankenhauses erzielen ließ [25]. Einschränkend muss jedoch angemerkt werden, dass die Voraussetzung für den Benefit unter NIV ein bestimmter Schweregrad der Erkrankung ist. So erklärt sich der fehlende Effekt der NIV im Vergleich zur medikamentösen Therapie bei COPD-Patienten mit einer leichtgradigen Form der ARI ( $\mathrm{pH}$ bei Beginn der NIV > 7,35) am ehesten dadurch, dass die Patienten nicht krank genug waren [12].

\section{Hypoxämische Verlaufsform der ARI}

Im Gegensatz hierzu ist die Datenlage bei der hypoxämischen Verlaufsform der ARI widersprüchlich. In einer frühen randomisierten Studie [45] war NIV der Kontrollgruppe nicht überlegen. Nur in einer Subgruppe bei Patienten mit $\mathrm{PCO}_{2}>45 \mathrm{~mm}$ $\mathrm{Hg}$ kam es infolge NIV zur Abnahme der Intubationsfrequenz, der Zeitdauer des Intensivaufenthalts und der Mortalität. In einer weiteren Studie zur ARI infolge außerhalb des Krankenhauses erworbener Pneumonie fand sich eine günstige Auswirkung der NIV auf die Intubationsfrequenz sowie Aufenthaltsdauer auf der Intensivstation [40]; einschränkend muss zu diesem Ergebnis jedoch gesagt werden, dass die Grundkrankheit eines großen Anteils der Patienten COPD war. Antonelli et al. konnten in 2 Studien zum hypoxischen Typ der ARI zeigen, dass es infolge der NIV zur Senkung der Intubationsfrequenz, der Komplikationsrate, der Mortalität kam und sich die Verweildauer auf der Intensivstation verringern ließ $[17,46]$.
In der Unterform der hypoxämisch verlaufenden ARI infolge des kardiopulmonalen Ödems (CPE), ergaben die randomisierten kontrollierten Studien mit CPAP (Druck 5-15 $\mathrm{H}_{2} \mathrm{O}$ ) eine Verbesserung von Vitalfunktion, Oxygenierung sowie Reduktion der Intubationsfrequenz [19,47]. Eine Entscheidungshilfe zwischen CPAP und PSV könnte sich bei gleichzeitigem Hinweis auf eine überlastete Atemmuskulatur ergeben. Es ließ sich nämlich zeigen, dass bei CPE und gleichzeitig nachweisbarer Hyperkapnie die mit einer zusätzlichen Entlastung der Atemmuskulatur verbundene PSV von Vorteil ist [48].

\section{NIV beim Weaning und zur Vermeidung der Reintubation}

In jüngerer Vergangenheit haben zwei randomisierte Studien bei Patienten mit COPD den Stellenwert der NIV nach invasiver Kurzzeitbeatmung von zwei bis sechs Tagen untersucht $[26,49]$. Die Patienten, die nach der invasiven Beatmungsphase nicht problemlos spontan atmen und damit extubiert werden konnten, wurden randomisiert in eine Gruppe, die weiter invasiv beatmet bzw. eine Gruppe, die extubiert und anschließend auf die NIV umgestellt wurde. In beiden Studien führte NIV zur Verkürzung der invasiven Beatmungszeit. Nur in der italienischen Studie zeigte sich eine Verbesserung der 3-Monats-Mortalität [26]. In diesem Zusammenhang bietet NIV möglicherweise die Option, bei Patienten, die nach Extubation erneut eine ventilatorische Insuffizienz entwickeln, eine Reintubation $\mathrm{zu}$ verhindern $[50,51]$. In einer retrospektiv kontrollierten Studie ergab sich, dass die Reintubationsphase durch NIV signifikant reduziert werden konnte [52]. Dieses Ergebnis konnte eine kürzlich veröffentlichte randomisierte kontrollierte Studie allerdings nicht bestätigen [53].

\section{NIV bei Patienten, die nicht intubiert werden sollen ("not to be intubated“)}

Mehrere retrospektive und unkontrollierte Studien der jüngeren Vergangenheit haben ergeben, dass NIV bei Patienten mit ARI, die aus unterschiedlichen Gründen nicht mehr intubiert werden sollen, durchaus praktikabel ist [38,54-57]. Vor allem im Sinne der verbesserten Lebensqualität ließen sich eine Reduktion der Dyspnoe und eine weitgehend erhaltene Autonomie erzielen. Dennoch bleiben ethische Fragen zu Grenzfällen mit dieser Indikation offen.

\section{Der postoperative Einsatz von NIV}

NIV in Form von CPAP und PSV wurde bei unterschiedlichen Indikationen postoperativ nach aorta-coronarer Bypasschirurgie, Bauchchirurgie, Transplantationschirurgie sowie Wirbelsäulenchirurgie vor allem zur Verbesserung physiologischer Parameter wie Gasaustausch, Parameter der Atemmechanik, extravaskulärem Lungenwasser eingesetzt [46,5866]. Demgegenüber wurden in diesen Studien Parameter bzgl. des klinischen Outcomes nicht berücksichtigt. Es ist in diesem Zusammenhang erwähnenswert, dass bei Patienten mit vorbestehender chronisch ventilatorischer Insuffizienz (CVI) bereits eine präoperative Adaptation an die NIV indiziert sein kann, um postoperative Komplikationen $\mathrm{zu}$ verhindern. $\mathrm{Zu}$ dieser Rubrik zählen z.B.: hyperkapnische Patienten vor Lungenvolumenreduktion, Skoliosechirurgie oder Lungentransplantation. 


\section{Zusammenfassung}

NIV in Ergänzung zur Standardtherapie führt bei der hyperkapnischen Verlaufsform der ARI zur Reduktion von Intubationsfrequenz und Komplikationsrate sowie zur Verbesserung der Mortalität. Demgegenüber bleibt die Datenlage zum Stellenwert der NIV bei hypoxämischer Verlaufsform der ARI widersprüchlich.

NIV führt zur Verkürzung der Entwöhnungsdauer und kann in Untergruppen die Reintubationsrate reduzieren. Bei Patienten, die nicht intubiert werden sollen, kann NIV zur Verbesserung der Lebensqualität beitragen. Postoperativ führt NIV zur Verbesserung der physiologischen Parameter, wobei die Auswirkung auf die klinischen Outcomedaten unklar bleibt.

\section{Empfehlung für zukünftige Studien}

Bei der hyperkapnischen Verlaufsform der ARI müssen die bisher (in den randomisierten Studien) geltenden Ausschlusskriterien kritisch überprüft werden, um möglicherweise weitere Patientengruppen der NIV zugänglich zu machen.

In weiteren Studien muss weiter abgeklärt werden, welcher Patient mit akut exazerbierter COPD „nicht krank genug“ bzw. „zu krank“ für die NIV ist.

Insbesondere bei der hypoxämischen Verlaufsform der ARI (d.h. bei Nicht-COPD-Patienten) werden weitere randomisierte kontrollierte Studien benötigt, um die Indikation zur NIV besser stellen zu können.

Zur CPE werden evidenzbasierte Kriterien bzgl. Differenzialtherapie zwischen CPAP und der Kombination aus PSV plus CPAP benötigt.

$\mathrm{Zu}$ den Bereichen „NIV bei Weaning vom Respirator, zur Vermeidung der Reintubationsrate und im postoperativen Verlauf“ werden Studien mit den Endpunkten „klinischer Outcome“ sowie „cost-effectiveness“ benötigt.

Es müssen Kriterien sowohl zur Anwendungsdauer der NIV pro Tag in der kritischen Initialphase der ARI als auch zum „weaning von NIV“ nach Stabilisierung der ARI gefunden werden.

\section{Literatur}

${ }^{1} \mathrm{NIH}$. Guidelines for the selection and management of consensus development conferences. In: 1995: Office of medical application of research. 1995

${ }^{2}$ Carlet J, Artigas A, Bihari D et al. The first European consensus conference in intensive care medicine: introductory remarks. Intensive Care Med 1992; 18: 180-181

${ }^{3}$ Simonds AK, Elliott MW. Outcome of domicilliary nasal intermittent positive pressure ventilation in restrictive and obstructive disorders. Thorax 1995; 50: 604-609

${ }^{4}$ Plant PK, Owen JL, Elliott MW. One year period prevalence study of respiratory acidosis in acute exacerbations of COPD: implications for the provision of non-invasive ventilation and oxygen administration. Thorax 2000; 55 (7): 550-554
${ }^{5}$ Richard JC, Carlucci A, Wysocki M et al. French multicenter survey: noninvasive versus conventional mechanical ventilation. Am J Respir Crit Care Med 1999; 159: A367

${ }^{6}$ Doherty MJ, Greenstone MA. Survey of non-invasive ventilation (NIPPV) in patients with acute exacerbations of chronic obstructive pulmonary disease (COPD) in the UK. Thorax 1998; 53: $863-867$

${ }^{7}$ Girault C, Richard JC, Chevron V et al. Comparative physiologic effects of noninvasive assist-control and pressure support ventilation in acute hypercapnic respiratory failure. Chest 1997; 111 (6): $1639-1648$

${ }^{8}$ Appendini L, Patessio A, Zanaboni S et al. Physiologic effects of positive end-expiratory pressure and mask pressure support during exacerbations of chronic obstructive pulmonary disease. Am J Respir Crit Care Med 1994; 149: 1069-1076

${ }^{9}$ Brochard L, Mancebo J, Wysocki M et al. Noninvasive ventilation for acute exacerbations of chronic obstructive pulmonary disease. N Engl J Med 1995; 333 (13): $817-822$

${ }^{10}$ Girault C, Chevron V, Richard JC et al. Physiological effects and optimisation of nasal assist-control ventilation for patients with chronic obstructive pulmonary disease in respiratory failure. Thorax 1997; 52 (8): 690-696

${ }^{11}$ Vitacca M, Clini E, Pagani M, Bianchi L, Rossi A, Ambrosino N. Physiologic effects of early administered mask proportional assist ventilation in patients with chronic obstructive pulmonary disease and acute respiratory failure. Crit Care Med 2000; 28 (6): $1791-1797$

12 Barbé F, Togores B, Rubi M, Pons S, Maimo A, Agusti AG. Noninvasive ventilatory support does not facilitate recovery from acute respiratory failure in chronic obstructive pulmonary disease. Eur Respir J 1996; 9 (6): 1240-1245

${ }^{13}$ Bott J, Baudouin SV, Moxham J. Nasal intermittent positive pressure ventilation in the treatment of respiratory failure in obstructive sleep apnoea. Thorax 1991; 46 (6): 457-458

${ }^{14}$ Kramer N, Meyer TJ, Meharg J, Cece RD, Hills NS. Randomized, prospective trial of noninvasive positive pressure ventilation in acute respiratory failure. Am J Respir Crit Care Med 1995; 151 (6): $1799-1806$

${ }^{15}$ Celikel T, Sungur M, Ceyhan B, Karakurt S. Comparison of noninvasive positive pressure ventilation with standard medical therapy in hypercapnic acute respiratory failure. Chest 1998; 114 (6): $1636-1642$

${ }^{16}$ Brochard L, Isabey D, Piquet J et al. Reversal of acute exacerbations of chronic obstructive lung disease by inspiratory assistance with a face mask. N Engl J Med 1990; 323 (22): 1523 1530

${ }^{17}$ Antonelli M, Conti G, Rocco M et al. A comparison of noninvasive positive-pressure ventilation and conventional mechanical ventilation in patients with acute respiratory failure. N Engl J Med 1998; 339 (7): 429-435

18 Patrick W, Webster K, Ludwig L, Roberts D, Wiebe P, Younes M. Noninvasive positive-pressure ventilation in acute respiratory distress without prior chronic respiratory failure. Am J Respir Crit Care Med 1996; 153 (3): 1005-1011

${ }^{19}$ Bersten AD, Holt AW, Vedig AE, Skowronski GA, Baggoley CJ. Treatment of severe cardiogenic pulmonary edema with continuous positive airway pressure delivered by face mask. $\mathrm{N}$ Engl J Med 1991; 325 (26): $1825-1830$

${ }^{20}$ Lin M, Yang YF, Chiang HT, Chang MS, Chiang BN, Cheitlin MD. Reappraisal of continuous positive airway pressure therapy in acute cardiogenic pulmonary edema. Short-term results and long-term follow-up. Chest 1995; 107 (5): 1379-1386

${ }^{21}$ Mehta S, Jay GD, Woolard RH et al. Randomized, prospective trial of bilevel versus continuous positive airway pressure in acute pulmonary edema. Crit Care Med 1997; 25 (4): 620-628 
${ }^{22}$ Vaisanen IT, Rasanen J. Continuous positive airway pressure and supplemental oxygen in the treatment of cardiogenic pulmonary edema. Chest 1987; 93 (3): $481-485$

23 Jounieaux V, Dury A, Delguste P, Rodenstein D. Effects of nasal positive pressure hyperventilation on the glottis in normal awake subjects. J Appl Physiol 1995; 79: 176 - 185

${ }^{24}$ Jounieaux V, Parreira VF, Delguste P, Aubert G, Rodenstein DO. Nasal mask pressure waveform and inspiratory muscle rest during nasal assisted ventilation. Am J Respir Crit Care Med 1997; 155 (6): 2096 - 2101

${ }^{25}$ Keenan SP, Kernerman PD, Cook DJ, Martin CM, McCormack D, Sibbald WJ. Effect of noninvasive positive pressure ventilation on mortality in patients admitted with acute respiratory failure: a meta-analysis. Crit Care Med 1997; 25 (10): 1685-1692

${ }^{26}$ Nava S, Ambrosino N, Clini E et al. Noninvasive mechanical ventilation in the weaning of patients with respiratory failure due to chronic obstructive pulmonary disease. A randomized, controlled trial. Ann Intern Med 1998; 128 (9): 721 - 728

${ }^{27}$ Bunburaphong T, Imanaka H, Nishimura M, Hess D, Kacmarek RM. Performance characteristics of bilevel pressure ventilators: a lung model study. Chest 1997; 111 (4): 1050-1060

${ }^{28}$ Aslanian P, El Atrous S, Isabey D et al. Effects of flow triggering on breathing effort during partial ventilatory support. Am J Respir Crit Care Med 1998; 157 (1): 135-143

${ }^{29}$ Bonmarchand G, Chevron V, Menard JF et al. Effects of pressure ramp slope values on the work of breathing during pressure support ventilation in restrictive patients [published erratum appears in Crit Care Med 1999 Jul; 27 (7): 1404]. Crit Care Med 1999; 27 (4): $715-722$

${ }^{30}$ Lofaso F, Aslanian P, Richard JC et al. Expiratory valves used for home devices: experimental and clinical comparison. Eur Respir J 1998; 11 (6): $1382-1388$

31 Ferguson GT, Gilmartin M. CO2 rebreathing during BiPAP ventilatory assistance. Am J Respir Crit Care Med 1995; 151: $1126-1135$

32 Younes M. Proportional assist ventilation, a new approach to ventilatory support. Theory. Am Rev Respir Dis 1992; 145 (1): $114-120$

${ }^{33}$ Ranieri VM, Grasso S, Mascia L et al. Effects of proportional assist ventilation on inspiratory muscle effort in patients with chronic obstructive pulmonary disease and acute respiratory failure. Anesthesiology 1997; 86 (1): 79-91

${ }^{34}$ Gay PC, Hess D, Hill NS. Randomized prospective trial of noninvasive pressure support ventilation and proportional pressure ventilation to treat acute respiratory insufficiency. Am J Respir Crit Care Med 1999; 159: A14

${ }^{35}$ Meecham Jones DJ, Paul EA, Grahame-Clarke C, Wedzicha JA. Nasal ventilation in acute exacerbations of chronic obstructive pulmonary disease: effect of ventilator mode on arterial blood gas tensions. Thorax 1994; 49 (12): 1222 - 1224

${ }^{36}$ Navalesi P, Fanfulla F, Frigerio P, Gregoretti C, Nava S. Physiologic Evaluation of Noninvasive Mechanical Ventilation Delivered with Three Types of Mask in Patients with Chronic Hypercapnic Respiratory Failure. Crit Care Med 2000; 28 (6): 1785-1790

${ }^{37}$ Vitacca M, Rubini F, Foglio K, Scalvini S, Nava S, Ambrosino N. Non-invasive modalities of positive pressure ventilation improve the outcome of acute exacerbations in COLD patients. Intensive Care Med 1993; 19 (8): 450-455

${ }^{38}$ Soo Hoo GW, Santiago S, Williams AJ. Nasal mechanical ventilation for hypercapnic respiratory failure in chronic obstructive pulmonary disease: determinants of success and failure. Crit Care Med 1994; 22 (8): 1253-1261

${ }^{39}$ Chevrolet JC, Jolliet P, Abajo B, Toussi A, Louis M. Nasal positive pressure ventilation in patients with acute respiratory failure. Difficult and time-consuming procedure for nurses. Chest 1991; 100 (3): $775-782$
${ }^{40}$ Confalonieri M, Potena A, Carbone G, Porta RD, Tolley EA, Umberto Meduri G. Acute respiratory failure in patients with severe community-acquired pneumonia. A prospective randomized evaluation of noninvasive ventilation. Am J Respir Crit Care Med 1999; 160 (5 Pt 1): 1585-1591

${ }^{41}$ Nava S, Evangelisti I, Rampulla C, Compagnoni ML, Fracchia C, Rubini F. Human and financial costs of noninvasive mechanical ventilation in patients affected by COPD and acute respiratory failure. Chest 1997; 111 (6): $1631-1638$

${ }^{42}$ Wood KA, Lewis L, Von Harz B, Kollef MH. The use of noninvasive positive pressure ventilation in the emergency department: results of a randomized clinical trial. Chest 1998; 113 (5): 13391346

${ }^{43}$ Schönhofer B. Respiratory high-dependency units in Germany. Monaldi Arch Chest Dis 1999; 54 (5): 448 - 451

${ }^{44}$ Plant PK, Owen JL, Elliott MW. Early use of non-invasive ventilation for acute exacerbations of chronic obstructive pulmonary disease on general respiratory wards: a multicentre randomised controlled trial. Lancet 2000; 355: 1931 - 1935

${ }^{45}$ Wysocki M, Tric L, Wolff MA, Millet H, Herman B. Noninvasive pressure support ventilation in patients with acute respiratory failure. A randomized comparison with conventional therapy. Chest 1995; 107 (3): $761-768$

${ }^{46}$ Antonelli M, Conti G, Bufi M et al. Noninvasive ventilation for treatment of acute respiratory failure in patients undergoing solid organ transplantation: a randomized trial. JAMA 2000; 283 (2): $235-241$

${ }^{47}$ Rasanen J, Vaisanen IT, Heikkila J, Nikki P. Acute myocardial infarction complicated by left ventricular dysfunction and respiratory failure. The effects of continuous positive airway pressure. Chest 1985; 87 (2): $158-162$

${ }^{48}$ Rusterholtz T, Kempf J, Berton C et al. Noninvasive pressure support ventilation (NIPSV) with face mask in patients with acute cardiogenic pulmonary edema (ACPE). Intensive Care Med 1999; 25 (1): $21-28$

${ }^{49}$ Girault C, Daudenthun I, Chevron V, Tamion F, Leroy J, Bonmarchand G. Noninvasive ventilation as a systematic extubation and weaning technique in acute-on-chronic respiratory failure: a prospective, randomized controlled study. Am J Respir Crit Care Med 1999; 160 (1): 86-92

${ }^{50}$ Schönhofer B, Sonneborn M, Haidl P, Kemper K, Köhler D. Stellenwert der intermittierenden Selbstbeatmung nach Entwöhnung vom Respirator. Pneumologie 1995; 49 (12): 689-694

51 Schönhofer B, Haidl P, Kemper P, Köhler D. Entwöhnung vom Respirator („Weaning“) bei Langzeitbeatmung. Dtsch Med Wochenschr 1999; 124: 1022 - 1028

${ }^{52}$ Hilbert G, Gruson D, Portel L, Gbikpi-Benissan G, Cardinaud JP. Noninvasive pressure support ventilation in COPD patients with postextubation hypercapnic respiratory insufficiency. Eur Respir J 1998; 11 (6): 1349-1353

53 Jiang JS, Kao SJ, Wang SN. Effect of early application of biphasic positive airway pressure on the outcome of extubation in ventilator weaning. Respirology 1999; 4 (2): 161 - 165

${ }^{54}$ Benhamou D, Girault C, Faure C, Portier F, Muir JF. Nasal mask ventilation in acute respiratory failure. Experience in elderly patients. Chest 1992; 102 (3): 912 - 917

${ }^{55}$ Meduri GU, Fox RC, Abou-Shala N, Leeper KV, Wunderink RG. Noninvasive mechanical ventilation via face mask in patients with acute respiratory failure who refused endotracheal intubation. Crit Care Med 1994; 22 (10): 1584-1590

${ }^{56}$ Meduri GU, Turner RE, Abou-Shala N, Wunderink R, Tolley E. Noninvasive positive pressure ventilation via face mask. Firstline intervention in patients with acute hypercapnic and hypoxemic respiratory failure. Chest 1996; 109 (1): 179-193 
57 Schönhofer B, Köhler D. Therapeutische Strategien der ventilatorischen Insuffizienz bei amyotropher Lateralsklerose. Nervenarzt 1998; 69 (4): 312 - 319

58 Aguilo R, Togores B, Pons S, Rubi M, Barbe F, Agusti AG. Noninvasive ventilatory support after lung resectional surgery. Chest 1997; 112 (1): $117-121$

${ }^{59}$ Gust R, Gottschalk A, Schmidt H, Bottiger BW, Martin E. Effects of continuous (CPAP) and bi-level positive airway pressure (BiPAP) on extravascular lung water after extubation of the trachea in patients following coronary artery bypass grafting. Intensive Care Med 1996; 22 (12): 1345-1350

${ }^{60}$ Kilger E, Briegel J, Haller M et al. Effects of noninvasive positive pressure ventilatory support in non-COPD patients with acute respiratory insufficiency after early extubation. Intensive Care Med 1999; 25 (12): 1374-1380

${ }^{61}$ Joris JL, Sottiaux TM, Chiche JD, Desaive CJ, Lamy ML. Effect of bilevel positive airway pressure (BiPAP) nasal ventilation on the postoperative pulmonary restrictive syndrome in obese patients undergoing gastroplasty. Chest 1997; 111 (3): 665-670

62 Jousela I, Rasanen J, Verkkala K, Lamminen A, Makelainen A, Nikki P. Continuous positive airway pressure by mask in patients after coronary surgery. Acta Anaesthesiol Scand 1994; 38 (4): $311-316$

${ }^{63}$ Matte P, Jacquet L, Van Dyck M, Goenen M. Effects of conventional physiotherapy, continuous positive airway pressure and non-invasive ventilatory support with bilevel positive airway pressure after coronary artery bypass grafting. Acta Anaesthesiol Scand 2000; 44 (1): 75-81

${ }^{64}$ Norregaard O, Jensen TM, Vindelev P. Effects of inspiratory pressure support on oxygenation and central haemodynamics in the normal heart during the postoperative period. Respir Med 1996; 90 (7): $415-417$

${ }^{65}$ Pinilla JC, Oleniuk FH, Tan L et al. Use of a nasal continuous positive airway pressure mask in the treatment of postoperative atelectasis in aortocoronary bypass surgery. Crit Care Med 1990; 18 (8): $836-840$

${ }^{66}$ Stock MC, Downs JB, Gauer PK, Alster JM, Imrey PB. Prevention of postoperative pulmonary complications with CPAP, incentive spirometry, and conservative therapy. Chest 1985; 87 (2): 151 157

Dr. med. Bernd. Schönhofer

Krankenhaus Kloster Grafschaft

Zentrum für Pneumologie und Allergologie

57392 Schmallenberg

E-mail: Bernd.Schoenhofer@t-online.de

\section{BUCHBESPRECHUNG}

F. Petermann: Asthma und Allergie. Verhaltensmedizinische Grundlagen und Anwendungen. (F. Petermann, Hogrefe) 1997. 376 Seiten. ISBN 3-8017-09795

Das von F. Petermann herausgegebene Werk ist mit nur geringen Änderungen - eingefügt wurde ein Kapitel über Compliance - in zweiter Auflage erschienen. Es ist weiterhin von hoher Aktualität.

Das Buch ist etwa zu gleichen Teilen den Themen „Asthma“ und „Allergische Hauterkrankungen“ gewidmet. Da sich die Verhaltensmuster der Patienten und die Behandlungsstrategien ähneln, lag es nahe, beide Krankheitsbilder in einem Band darzustellen.

Bei der Behandlung chronischer Erkrankungen ist es mit der korrekten Verordnung von Medikamenten nicht getan. Langfristig sind gute Erfolge nur von einem interdisziplinären Therapiekonzept zu erwarten. Eine fruchtbare Zusammenarbeit zwischen Ärzten, Psychologen, Pädagogen und Physiotherapeuten setzt aber gegenseitiges Verständnis voraus. Die Vertreter verschiedener Disziplinen brauchen ein Basiswissen in allen Fachgebieten, um die unterschiedlichen Denk- und Redeweisen kennenzulernen. Das vorliegende Buch ist diesem Anliegen gewidmet.

Neben den medizinischen Grundlagen und neuen Erkenntnissen der Verhaltensmedizin werden in mehreren Beiträgen Inhalte und didaktische Konzepte der Patientenschulung beschrieben und auch die geeigneten Arbeitsmaterialien präsentiert. In den letzten Jahren ist zunehmend deutlich geworden, dass nur der informierte und trainierte Patient in der Lage ist, Eigenverantwortung zu übernehmen und zu einem günstigen Krankheitsverlauf aktiv beizutragen.

Es ist dem Herausgeber gelungen, die relevanten Themen zu formulieren und für jedes Kapitel die geeigneten Autoren zu gewinnen. Das Buch ist informativ und gut lesbar. Es vermittelt die für eine Teamarbeit notwendige Synopsis unterschiedlicher therapeutischer Konzepte. Wer an der medizinischen, psychologischen oder pädagogischen Betreuung von Patienten mit Asthma oder Neurodermitis interessiert und beteiligt ist, wird dieses Buch mit Gewinn zur Hand nehmen. 